PAPER

\title{
Neuroanatomical localisation and clinical correlates of white matter lesions in the elderly
}

\author{
S Artero, H Tiemeier, N D Prins, R Sabatier, M M B Breteler, K Ritchie
}

J Neurol Neurosurg Psychiatry 2004;75:1304-1308. doi: 10.1136/jnnp.2003.023713

See end of article for authors' affiliations

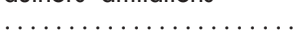

Correspondence to: Dr S Artero, INSERM, EMI 0361, Hôpital la Colombière, Pavillon 42, 39 avenue Charles Flahault 34093 Montpellier cedex 5, France; artero@ montp.inserm.fr

Received 17 July 2003

Revised 24November 2003

Accepted

17 December 2003
Background: White matter lesions (WML) in elderly people co-occur with hypertension, depression, and cognitive impairment. Little is known about the density and distribution of WML in normal elderly people, whether they occur randomly in the aging brain or tend to cluster in certain areas, or whether patterns of WML aggregation are linked to clinical symptoms.

Objectives: To describe patterns of WML distribution in a large representative population of elderly people using non-inferential cluster analysis; and to determine the extent to which such patterns are associated with clinical symptomatology.

Method: A population sample of 1077 elderly people was recruited. Multiple analysis of correspondence followed by automatic classification methods was used to explore overall patterns of WML distribution. Correspondence was then sought between these patterns and a range of cerebrovascular, psychiatric, and neurological symptoms.

Results: Three distinct patterns of spatial localisation within the brain were observed, corresponding to distinct clusters of clinical symptoms. In particular WML aggregation in temporal and occipital areas was associated with greater age, hypertension, late onset depressive disorder, poor global cognitive function, and overall WML frequency.

Conclusions: WML localisation is not random in the aging brain, and their distribution is associated with age and the presence of clinical symptoms. Age differences suggest there may be patterns of progression across time; however, this requires confirmation from longitudinal imaging studies.
W hite matter lesions (WML) in elderly people result from degenerative changes in small vessels, ${ }^{12}$ with hypertension and arteriosclerosis being identified as significant risk factors. ${ }^{4}$ While WML are known to be common in the elderly, ${ }^{5}$ magnetic resonance imaging (MRI) studies have shown higher rates in elderly people with depression than in age matched controls. ${ }^{78}$ This is particularly true of late onset depression, ${ }^{9}{ }^{10}$ an observation that has led to the notion of "vascular depression." Other studies have suggested a relation between WML and dementia. ${ }^{11-13}$ Several investigators have proposed that localisation may be important, with frontal lesions being associated with depression ${ }^{14} 15$ and periventricular lesions being implicated in cognitive impairment. ${ }^{12}{ }^{16}$ Most studies have been based on a global estimate of lesion volume, or alternatively on the presence or absence of WML.

A further limitation has been the use of a "top down" approach, in which lesion frequency is explored in contrasting clinical cohorts and controls. Such an approach does not take into account the heterogeneity of clinical syndromes and the possibility that WML clusters may be related to specific symptoms cutting across disease categories. Moreover little is known about patterns of WML distribution in the normal brain. Are they randomly distributed or do they aggregate in certain areas? Are there clustering patterns unique to certain clinical profiles or are they simply an exaggeration of patterns seen in normal elderly people?

In the present study, we took an alternative "bottom up" approach to the question of WML and brain aging by starting with the question of how WML are spatially distributed in elderly people, and whether patterns can be identified that may ultimately be linked to clinical features. The originality of this approach is that instead of comparing WML density in different case and control groups, as in previous research, we have explored a staging model—-that is, can we see a pattern in both location and density? And if so, how do such patterns or stages relate to the appearance of clinical symptoms? In order to establish the possible existence of a general trend in WML progression, we used multidimensional data analysis to establish patterns of spatial distribution in a large representative population of elderly persons, and attempted to link such patterns to the type and severity of symptom clusters.

\section{METHODS}

The subjects for the present analysis are participants in the Rotterdam scan study, which was designed to explore determinants and consequences of age related brain abnormalities in the elderly. In 1995 and 1996, 1904 subjects were randomly selected from within each five year age group between 60 and 90 years of age with an equal contribution of both sexes from two large ongoing population based studies. ${ }^{17}{ }^{18}$ Among the selected subjects, 187 were not eligible because of dementia, MRI contraindications, or blindness, and so were excluded from our study. Of the 1717 eligible people, 640 refused. Thus in all, 1077 nondemented elderly individuals participated in the study (overall response 63\%). We screened all participants for dementia using the mini-mental state examination (MMSE) and the geriatric mental state schedule. Participants who were positive at screening underwent additional cognitive testing with the Cambridge mental disorders of the elderly examination. People who were then thought to have dementia were excluded.

Informed consent was obtained from all participants and the study design was approved by the medical ethics committee of the Erasmus Medical Centre, Rotterdam.

Abbreviations: CESD, Center of Epidemiologic Studies depression scale; MCA, multiple correspondence analysis; MMSE, mini-mental state examination; WML, white matter lesion 


\section{MRI procedure}

Cranial MRI scanning was undertaken in all 1077 participants. We carried out axial T1, T2, and proton density weighted scans on 1.5 Tesla MRI scanners (MR Gyroscan, Philips, Best, Netherlands, and MR VISION, Siemens, Erlangen, Germany). The slice thickness was 5 or $6 \mathrm{~mm}$ (scanner dependent), with an interslice gap of $20 \%$. Laser hard copies were printed with a reduction factor of 2.7. White matter lesions were considered present if visible as hyperintense on proton density and T2 weighted images, without corresponding prominent hypointensity on $\mathrm{Tl}$ weighted images. We considered white matter lesions to be periventricular if they were directly adjacent to the ventricle; otherwise we considered them as subcortical.

White matter lesion severity was graded for periventricular and subcortical areas separately. In summary, periventricular white matter lesions were scored semiquantitatively (range 0 to 9). Subcortical WML were categorised according to their maximum diameter (on hard copy) as small (1-3 mm), medium $(3-10 \mathrm{~mm})$, or large $(>10 \mathrm{~mm})$. The number of subcortical WML was rated per size category for the frontal, parietal, occipital, and temporal lobes (range 0 to 29.5). ${ }^{4}$ We approximated a total subcortical WML volume (in millimetres on hardcopy) by assuming subcortical WML are spherical with diameters of 2,6 , or $12 \mathrm{~mm}$ (according to their size category) and adding these volumes.

Atrophic change was scored on Tl weighted images. A distinction was made between cortical and subcortical brain atrophy. Cortical atrophy was rated on a semiquantitative scale (range 0 to 15) using reference scans. Subcortical atrophy was measured by the ventricle to brain ratio (range 0.21 to 0.45$).{ }^{19}$ The ventricle to brain ratio is the ratio between the biventricular width and the width of the brain at the same level.

\section{Clinical variables}

Analyses included age (as a continuous variable), sex, and educational status (highest education level obtained) according to UNESCO. ${ }^{20}$ The clinical variables selected from the medical examination were those most commonly linked to vascular risk, namely hypertension (defined as a systolic blood pressure of $160 \mathrm{~mm} \mathrm{Hg}$ or over, or a diastolic blood pressure of $95 \mathrm{~mm} \mathrm{Hg}$ or over, or the use of blood pressure lowering drugs); a random or post-load serum glucose concentration of $>11 \mathrm{mmol} / \mathrm{l}$; smoking (divided into current smokers and non-smokers); and a history of stroke, validated by reference to medical records and verified by a neurologist. ${ }^{21}$ Global cognitive function was measured by the MMSE. ${ }^{22}$ Information was also obtained on any history of depressive episodes, the age of onset, and treatment. Depressive symptoms were assessed using the Center of Epidemiologic Studies depression scale (CESD). ${ }^{23}$ A depressive disorder was defined in two ways: first, when the subject scored at or above 16 points on the CESD; and second, if the episode had required treatment from a general practitioner, psychologist, or psychiatrist. We divided the age of onset at the cut off point of 60 years, in keeping with previous studies. ${ }^{24} 25$

\section{Statistical analysis}

Multidimensional data analysis was used to show patterns of lesion development independently of aetiological hypotheses. This method detects patterns such as grouping and opposition and eliminates "noise" caused by outliers. We used multiple correspondence analysis (MCA ${ }^{26}$ to provide a graphic representation of WML distribution in the brain. The analysis was carried out with 1077 subjects and 20 variables representing periventricular and subcortical lesions (frontal, temporal, parietal, and occipital), divided into quintiles (five groups) according to severity as determined by reference to size and density of lesions. With this analysis we retained four axes, which explained most of the variation, and suppressed non-interpretable axes (noise). Three distinct patterns of WML were obtained by MCA.

With the four principal axes we undertook a classification with an ascending hierarchy (cluster analysis) using Ward's criteria. These clusters were depicted by the method of convex envelopes. In this way, clusters of individual subjects can be visualised and superimposed on the graphical representation of WML distribution. In all, three clusters could be defined corresponding to the three patterns in the MCA. Subsequently, the individual subjects who belong to a spatially determined group were identified. This permits a general portrait of each group by reference to vascular, cognitive, psychiatric, and neurological features. Next, we tested statistically whether the clusters of individuals-based solely on the distribution of brain lesions-differed with regard to the clinical features. Significance testing was carried out for qualitative variables using a $\chi^{2}$ test, and for quantitative variables using analysis of variance or the Kruskal-Wallis test, depending on the normality of the distribution.

Multidimensional statistical analyses were carried out using the ADE-4 program $^{27}$ and univariate analyses with the SPSS (Statistical Package for Social Science) program, version 11.0.

\section{RESULTS}

MCA analysis showed a non-random pattern of WML distribution and produced three distinct anatomical clusters of white matter lesions within this elderly cohort. Figure 1 presents a two dimensional representation of the results of the MCA. The first pattern is characterised essentially by a small number of WML (quintile 1), which are confined principally to frontal, parietal, and periventricular regions. The second pattern is one of increasing WML frequency (quintiles 2, 3, and 4) in the frontal, parietal, and periventricular areas. Finally, the third clearly discernable pattern is of very high density WML (quintile 5) within frontal, parietal, and periventricular areas, also extending to occupy the occipital and temporal regions.

Cluster analysis was then applied to the four axes retained by the MCA. Three groups of patients were defined, corresponding to patterns of WML distribution. With this analysis, subjects belonging to the three spatially determined cluster were identified. This allowed us to determine the cognitive, psychiatric, and neurological features that characterise the pattern of lesions independently of clinical diagnostic category. Figure 2 is a visual representation of these three groups that should be interpreted by superimposition on fig 1 . In other words, fig 1 shows the pattern of the WML variables, while fig 2 shows the corresponding clustering of the individuals.

The clinical characteristics of the three groups are given in table 1 . No significant differences were found in the sex ratio, level of education, percentage of smokers, or diabetic subjects across the three groups. On the other hand, mean age increased significantly from group 1 to group $3(\mathrm{p}<0.001)$. An increase was also observed from group 1 to group 3 in the number of subjects with depression, in particular those with late onset depression $(\mathrm{p}<0.05)$, and with hypertension $(p<0.001)$. Overall the three clinical groups can easily be distinguished in relation to WML distribution.

\section{Group 1}

This group, corresponding to WML pattern 1, was characterised by a very small number of lesions confined to frontal, parietal, and periventricular areas. This group was the 
Axis 2

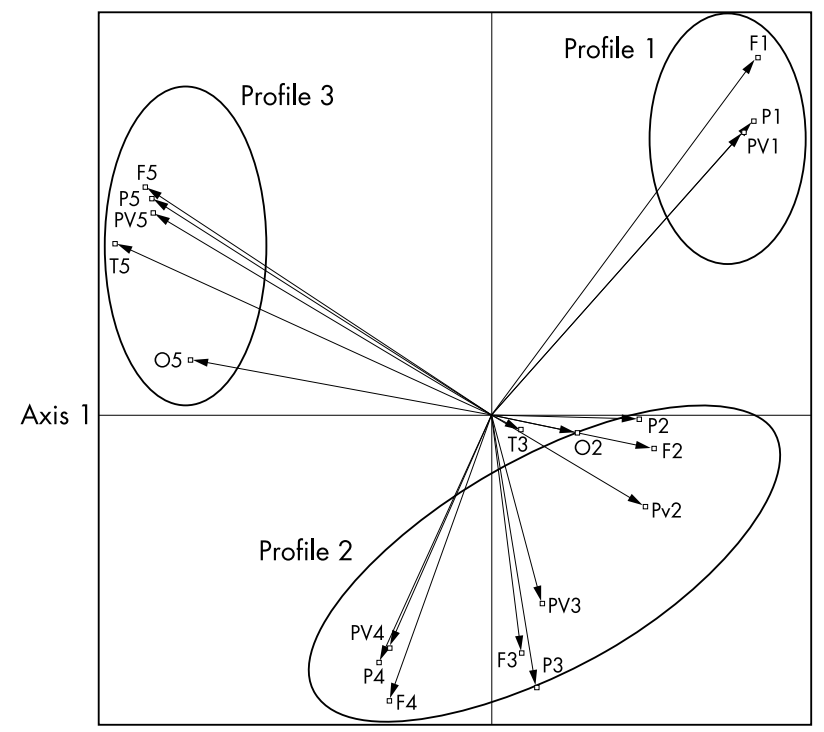

Figure 1 Patterns of spatial localisation of white matter lesions in the elderly population. $F$, frontal lesions; $O$, occipital lesions; $P$, parietal lesions; PV, periventricular lesions; T, temporal lesions. 1, 2, 3, 4, 5: severity of WML in quintiles (five groups). Note that in this two dimensional figure only two of the four retained axes (Nos 1 and 2) can be shown.

youngest with a mean age of $68.1(5.7)$ years $(\mathrm{p}<0.001)$. The mean MMSE score was 27.9 (2.1). This group had a very small proportion of subjects with a positive CESD score $(2.6 \%)$ - a rate significantly lower than either of the other two groups $(\mathrm{p}<0.05)$. Examining specific item responses to the CESD showed that only dimensions 1 and 2 (depressed affect and positive affect) were lower than in the other groups $(\mathrm{p}<0.01)$, but not dimensions 3 and 4 (somatic, motor retardation and interpersonal difficulties).

\section{Group 2}

This group was superimposed over WML pattern 2, showing frontal, parietal, and periventricular lesions but at a much higher density than group 1. It may be considered an intermediate group with a similar mean MMSE score (27.5), although the subjects were slightly older (mean age, 71.7 (7.1) years).

\section{Group 3}

This clinical group corresponded most closely to the third WML pattern, with a very high density of WML lesions in frontal, parietal, and periventricular areas, extending into the occipital and temporal regions. This was the oldest group with a mean age of $77.2(7.0)$ years. The MMSE score (27.0) was lower than in the other two groups $(p<0.001)$ and the percentage of subjects with clinical depression was also much greater, at $11 \%(\mathrm{p}<0.05)$. The percentages with hypertension $(67.1 \%)$ and a history of stroke $(12.6 \%)$ were much higher than in either group 1 or group $2(\mathrm{p}<0.001)$. The third group also had more cortical atrophy $(7.1(3.1) \%, p<0.001)$ and higher rates of late onset depression (11.1\%) than either of the other groups $(\mathrm{p}<0.005)$.

\section{DISCUSSION}

In this analysis we have taken an alternative approach to the traditional inferential disease/WML location method by first examining overall patterns and density of WML distribution in neurologically defined parts of the brain in a representative population of elderly people, and then examining the relation

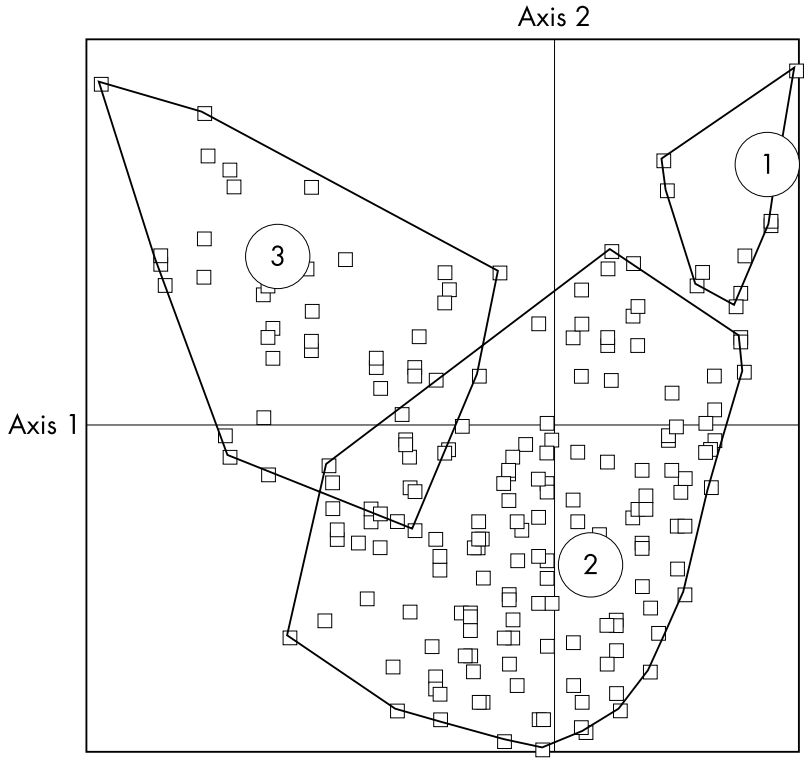

Figure 2 Visual representation of the cluster analysis. Note that in this two dimensional figure only two of the four retained axes (Nos 1 and 2) can be shown.

of these patterns to symptomatology rather than to disease entities. We found that in this elderly population, WML were not randomly distributed. Spatial analysis revealed three separate patterns of WML aggregation. which may be a useful means of classifying subjects in future studies, as these three groups appear to be associated with different clinical profiles. The first two showed low and intermediate levels of depressive symptomatology, while the third was characterised by high rates of depressive symptoms, cognitive impairment, and cerebrovascular risk factors. The results suggest the possible "vulnerability" of frontal areas to WML, in the sense that these areas are the first to show lesions in relatively symptom-free elderly people, and the lesions increase in density when other areas are affected. This may partly be explained by the architecture of the cerebral vascular system, as the arteries penetrate deep into the white matter in frontal areas.

Across the three groups, greater WML severity is associated with lower MMSE scores. Patterns of greater WML severity were also associated with an increased frequency of late onset depressive symptoms, although few subjects crossed the threshold into major depression, as defined by CESD scores. This feature was most marked in the third group where the highest CESD scores were associated with the presence of WML in the occipital and temporal areas. Clinically this was reflected in higher rates of negative and depressive affect, but not in somatic, interpersonal, and motor complaints. This group was also at greater vascular risk, had more cerebrovascular accidents, and had higher blood pressures and a greater degree of cortical atrophy. These findings are in support of a late onset depressive syndrome. In the case of both cognitive and affective symptomatology there was a high correlation with WML density, although relatively few subjects met diagnostic criteria for dementia and major depression, underlying the importance of examining WML in relation to symptomatology rather than to disease groups.

The three patterns of WML aggregation were also distinguished by age. High WML density and posterior localisation are clearly associated with greater age, raising the possibility of ordered progression of WML across time. It may be hypothesised from this cross sectional study that in the mid-60s small numbers of lesions are seen predominantly 
Table 1 Description of the three groups of elderly subjects detected by cluster analysis

\begin{tabular}{|c|c|c|c|c|}
\hline Variable & Group 1 & Group 2 & Group 3 & p Valuet \\
\hline Age (years) & $68.05(5.67)$ & $71.72(7.05)$ & $77.17(6.98)$ & 0.000 \\
\hline MMSE score & $27.85(2.11)$ & $27.47(2.04)$ & $26.96(2.63)^{*}$ & 0.000 \\
\hline CESD score & $4.84(4.80)$ & $5.93(6.34)$ & $6.77(6.54)$ & 0.007 \\
\hline \multicolumn{5}{|l|}{ Four dimensions of CESD } \\
\hline Dimension 1 (depressed affect) & $0.7(1.44)$ & $1.12(2.19)$ & $1.39(2.59)$ & 0.005 \\
\hline Dimension 2 (positive affect) & $1.73(2.31)$ & $2.25(2.63)$ & $2.62(2.89)$ & 0.003 \\
\hline \multicolumn{5}{|l|}{ Dimension 3 (somatic and } \\
\hline retarded activity) & $2.10(2.23)$ & $2.28(2.56)$ & $2.52(2.58)$ & 0.243 \\
\hline Dimension 4 (interpersonal) & $0.21(0.55)$ & $0.28(0.66)$ & $0.20(0.55)$ & 0.171 \\
\hline Cortical atrophy & $4.52(2.50)$ & $5.30(2.65)$ & $7.14(3.07)$ & 0.000 \\
\hline Subcortical atrophy & $0.30(0.02)$ & $0.31(0.36)$ & $0.32(0.03)$ & 0.000 \\
\hline \multicolumn{5}{|l|}{ White matter lesions } \\
\hline Frontal & $0.09(0.02)$ & $0.25(0.45)$ & $3.06(3.01)$ & 0.000 \\
\hline Parietal & $0.04(0.05)$ & $0.18(0.20)$ & $1.97(1.64)$ & 0.000 \\
\hline Temporal & $0(0.0)$ & $0.007(0.07)$ & $0.04(0.19)$ & 0.000 \\
\hline Occipital & $0(0.0)$ & $0.05(0.03)$ & $0.17(0.51)$ & 0.000 \\
\hline \multirow[t]{2}{*}{ Periventricular } & $0.34(0.73)$ & $1.98(1.51)$ & $5.39(1.80)$ & 0.000 \\
\hline & \multicolumn{2}{|c|}{ Per cent distribution } & & p Value \\
\hline Female sex & $48.4(93)$ & $50.8(334)$ & $57.2(127)$ & 0.154 \\
\hline Education $<8$ years & $12.6(24)$ & $11.9(78)$ & $9.5(21)$ & 0.558 \\
\hline Late onset depressive persons & $3.9(6)$ & $8.5(46)$ & $11.1(22)$ & 0.05 \\
\hline Positive CESD (score >16) & $2.6(5)$ & $7.7(50)$ & $11.0(24)$ & 0.05 \\
\hline CVA & $0.5(1)$ & $4.2(27)$ & $12.6(27)$ & 0.000 \\
\hline \multicolumn{5}{|l|}{ Cerebrovascular risk factors } \\
\hline $\begin{array}{l}\text { Hypertension or use of } \\
\text { antihypertensive drugs }\end{array}$ & $40.6(78)$ & $49.2(324)$ & 67.1 (149) & 0.000 \\
\hline Diabetes mellitus & $3.1(6)$ & $7.6(50)$ & $8.6(19)$ & 0.06 \\
\hline Current smoker & $16.7(32)$ & $18.2(120)$ & $13.6(30)$ & 0.288 \\
\hline \multicolumn{5}{|c|}{$\begin{array}{l}\text { Values are mean (SD), top, or } \%(\mathrm{n}) \text {, bottom. } \\
{ }^{*} \text { Group } 3 \text { significantly different from groups } 1 \text { and } 2 \text {. } \\
+ \text { Variance analysis or Kruskal-Wallis test as appropriate. } \\
\mp \chi^{2} \text { test. } \\
\text { CESD, Center of Epidemiologic Studies depression scale; CVA, cerebrovascular accident; MMSE, mini-mental state } \\
\text { examination. }\end{array}$} \\
\hline
\end{tabular}

in frontal areas but also to some extent in parietal and periventricular regions. In the early 70s lesions show a similar spatial distribution but are present in much greater numbers. In the late 70 s not only is there an even greater density in frontal, parietal, and periventricular areas, but the occipital and temporal areas are also involved. ${ }^{28}$ The presence of lesions in temporal and occipital areas may thus signal a greater severity of WML, with subclinical states crossing over into pathology. This is consistent with clinical observations that WML are localised in the occipital and temporal regions in neurodegenerative and psychiatric disorders. ${ }^{29-33}$ The clusters of WML derived from this analysis may provide a useful future classification system for differentiating patients and for monitoring.

Though the response rate in this study was reasonable, response bias should be taken into consideration. Participants from the study were younger than nonparticipants (mean age difference, 3.8 years; $p<0.001$ ) and participants originally invited from the Zoetermeer study had a lower blood pressure (age and sex adjusted difference, 2.4 $\mathrm{mm} \mathrm{Hg} ; \mathrm{p}=0.03$ ). Because old age and high blood pressure are established risk factors for the presence and severity of WML, the group with most severe WML is probably underrepresented in our study. Baseline MMSE scores could only be compared between participants and non-participants from the Rotterdam study, but it is likely that comparisons would be the same if we had been able to include the Zoetermeer study. Participants had higher baseline MMSE scores than non-participants (age and sex adjusted mean difference, 0.4 points; $\mathrm{p}<0.001$ ). Most probably, the selection can only have impeded the detection of patterns of spatial localisation of
WML corresponding to distinct clusters of clinical symptoms. Our findings would thus constitute a conservative estimation of the relation between age, WML localisation, and clinical symptoms.

A longitudinal general population study of WML is of course required to explore our tentative hypothesis of progression from frontal through parietal and finally to temporal and occipital regions. Multidimensional data analysis allows a non-hypothesis driven classification and therefore a more open descriptive approach to examining WML distribution, but does not prove causality. Longitudinal MRI studies that permit the construction of predictive models of pathology onset will allow us to determine whether this progression is an inevitable feature of aging or whether the extension of WML to posterior areas is caused by the superimposition of a disease process over "normal" brain aging. To our knowledge there are currently no imaging data available from general population studies that span the 20 year period necessary to map this type of progression.

\section{ACKNOWLEGEMENTS}

Supported by grants from the Netherlands Organisation for Scientific Research (NWO) and the Health Research and Development Council $(\mathrm{ZON})$.

\section{Authors' affiliations}

S Artero, K Ritchie, Institut National de la Santé et de la Recherche Médicale, INSERM EMI 0361, Epidemiology of Nervous System Pathologies, Montpellier, France

R Sabatier, Faculté de Pharmacie, Université de Montpellier I, Montpellier 
H Tiemeier, N D Prins, M M B Breteler, Department of Epidemiology and Biostatistics, Erasmus Medical Centre, Rotterdam, Netherlands

Competing interests: none declared

\section{REFERENCES}

1 Pantoni L, Garcia JH. Pathogenosis of leukoaraiosis: a review. Stroke 1997;28:652-9.

2 Roman GC, Erkinjuntti T, Wallin A, et al. Subcortical ischaemic vascular dementia. Lancet Neurol 2002;1:426-36.

3 Greenwald BS, Kramer-Ginsberg E, Krishnan KRR, et al. A controlled study of MRI signal hyperintensities in older depressed patients with and without hyperintensities. J Am Geriatr Soc 2001;49:1218-25.

4 de Leeuw FE, de Groot JC, Bots ML, et al. Carotid atherosclerosis and cerebral white matter lesions in a population based magnetic resonance study. I Neurol 2000;247:291-6.

5 Scheltens P, Barkhof F, Valk J, et al. White matters lesions on magnetic resonance imaging in clinically diagnosed Alzheimer's disease. Evidence for heterogeneity. Brain 1992;115:735-48.

6 Breteler MMB, van Swieten JC, Bots ML, et al. Cerebral white matter lesions, vascular risk factors, and cognitive function in a population in a populationbased study; the Rotterdam study. Neurology 1994;44:1246-52.

7 Hickie I, Scott E, Mitchell P, et al. Subcortical hyperintensities on magnetic resonance imaging: clinical correlates and prognostic significance in patients with severe depression. Biol Psychiatry 1995;37:151-60.

8 Greenwald BS, Kramer-Ginsberg E, Krishnan KRR, et al. MRI signal hyperintensities in geriatric depression. Am J Psychiatry 1996;153:1212-15.

9 O'Brien JT, Ames D, Schwietzer I. White matter changes in depression and Alzheimer's disease: a review of magnetic resonance studies. Int J Geriatr Psychiatry 1996;11:681-694.

10 Krishnan KR, Gadde KM. The pathophysiologic basis for late life depression: imaging studies of the aging brain. Am J Geriatr Psychiatry 1996;4(suppl 1):S226-33.

11 De Groot JC, de Leeuw FE, Oudkerk M, et al. Periventricular cerebral white matter lesions and cognitive function: the Rotterdam Scan Study. Ann Neurol 2000;47:145-51.

12 De Groot JC, de Leeuw FE, Oudkerk M, et al. Periventricular cerebral white matter lesions predict rate of cognitive decline. Ann Neurol 2002;52:335-41.

13 Kuller LH, Lopez OL, Newman A, et al. Risk factors for dementia in the cardiovascular Health Cognition Study. Neuroepidemiology 2003;22:13-22.

14 Krishnan KR. Organic bases of depression in the elderly. Annu Rev Med 1991;42:261-6.

15 Macfall JR, Payne ME, Provenzale JE, et al. Medial orbital frontal lesions in late-onset depression. Biol Psychiatry 2001;49:803-6.

16 Boone KB, Miller BL, Lesser IM, et al. Neuropsychological correlates of whitematter lesions in healthy elderly subjects. A threshold effect. Arch Neurol 1992;49:549-54.
17 Hofman A, Grobbee D, de Jong P, et al. determinants of disease and disability in the elderly: the Rotterdam Elderly Study. Eur J Epidemiol $1991 ; 7: 403-22$.

18 Hofman A, Boomsma F, Schalekamp M, et al. Raised blood pressure and plasma noradrenaline concentrations in teenagers and young adults selected from an open population. BMJ 1979;i:1536-8.

19 den Heijer T, Oudkerk M, Launer $\sqcup$, et al. Hippocampal, amygdalar, and global brain atrophy in different apolipoprotein E genotypes. Neurology 2002;59:746-748.

20 UNESCO. International standard classification of education (ISCED). Paris: UNESCO, 1976.

21 Vermeer SE, Koudstaal PJ, Oudkerk M, et al. Prevalence and risk factors of silent brain infarcts in the population-based Rotterdam Scan Study. Stroke 2002;33:21-5

22 Folstein MF, Folstein SE, McHugh PR. "Mini-mental state". A practical method for grading the cognitive state of patients for the clinician. J Psychiatr Res 1975;12:189-98.

23 Radloff LS. The CESD Scale: a self-report depression scale for research in the general population. Appl Psychol Meas 1977;1:385-401

24 Figiel GS, Krishnan KR, Doraiswamy PM, et al. Subcortical hyperintensities on brain magnetic resonance imaging: a comparison between late age onset and early onset elderly depressed subjects. Neurobiol Aging $1991 ; 12: 245-7$.

25 Salloway S, Malloy P, Kohn R, et al. MRI and neuropsychological differences in early- and late-life-onset geriatric depression. Neurology 1996;46:1567-74.

26 Saporta G. Probabilités, analyse des données et statistique. Technip 1990:493.

27 Thioulouse J, Chessel D, Dolédec S, et al. ADE-4: A multivariate analysis and graphical display software. Stat Comput 1997;7:75-83.

28 De Leeuw FE, de Groot JC, Achten E, et al. Prevalence of cerebral white matter lesions in elderly people: a population based magnetic resonance imaging study. The Rotterdam Scan Study. I Neurol Neurosurg Psychiatry $2001 ; 70: 9-14$

29 Bokde AL, Teipel SJ, Zebuhr Y, et al. A new rapid landmark-based regional MRI segmentation method of the brain. J Neurol Sci 2002;194:35-40.

30 Braak H, Griffing K, Braak E. Neuroanatomy of Alzheimer's disease. Alzheimer's Res 1997;3:235-47.

31 Bergmann M, Kuchelmeister K, Schmid KW, et al. Different variants of frontotemporal dementia: A neuropathological and immunohistochemical study. Acta Neuropathol 1996;92:170-9.

32 Yamanouchi H, Sugivira S, Tomonaga M. Decrease in nerve fibres in cerebral white matter in progressive subcortical vascular encephalopathy of Binswanger type. J Neurol 1989;236:382-7.

33 Erkinjuntti $T$, Benavente $O$, Eliaziw $M$, et al. Diffuse vacuolisation (spongiosis) and arteriolosclerosis in the frontal white matter occurs in vascular dementia. Arch Neurol 1996;53:325-32. 